\title{
Skyrmions in Bose-Einstein Condensates
}

\author{
Richard Battye ${ }^{1}$, Nigel Cooper ${ }^{2}$ and Paul Sutcliffe* \\ 1 Jodrell Bank Observatory, Macclesfield, Cheshire SK11 9DL U.K. \& Department of \\ Physics and Astronomy, Schuster Laboratory, University of Manchester, Brunswick St, \\ Manchester M13 9PL, U.K. \\ ${ }^{2}$ Theory of Condensed Matter Group, Cavendish Laboratory, Madingley Road, \\ Cambridge CB3 OHE, U.K. \\ ${ }^{*}$ Institute of Mathematics, University of Kent at Canterbury, Canterbury CT2 7NF, \\ U.K.

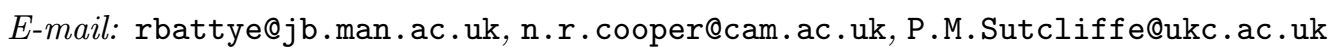

Abstract: We compute Skyrmion solutions of the Gross-Pitaevskii equations describing binary Bose-Einstein condensates and discuss parameter regimes in which they exist.

\section{Introduction}

Experimental advances in the formation and control of ultracold atomic gases are allowing detailed studies of the properties of Bose-Einstein condensates (BECs) with both single and multiple components. The condensate wavefunctions satisfy coupled Gross-Pitaevskii equations and these can have topological soliton solutions, providing an experimental setup in which the properties of various types of soliton may be studied. In the case of a single component the Gross-Pitaevskii equation has a vortex solution in two dimensions, in which the phase of the wavefunction changes by $2 \pi$ around a closed loop containing the vortex core. Extending such solutions into three space dimensions yields vortex strings which have been created experimentally and their properties studied in some detail ii]. Multiple component condensates have a much richer structure and in particular they offer the possibility of supporting truly three-dimensional solitons, such as monopoles [i]

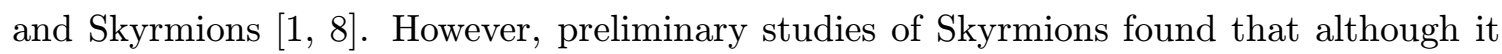
was possible to construct configurations with the topology of a Skyrmion there were no associated stationary solutions of the Gross-Pitaevskii equation - essentially a Skyrmion configuration was found to be unstable to collapse. Subsequently the present authors [2] found that stable Skyrmion solutions do exist, for particular parameter regimes, which do not include those used in the earlier studies but do include physically relevant situations.

${ }^{*}$ Speaker. 
Here we shall extend the results in [īin] by removing some of the approximations made there; in particular we include the total density as a dynamical degree of freedom. After reviewing the Gross-Pitaevskii equations we describe the topology of a Skyrmion solution and derive the requirement of phase separation before finally presenting the results of our numerical computations.

\section{The Gross-Pitaevskii equations}

For a binary BEC, that is, the wave function has two components $\psi_{\alpha}$ with $\alpha=1,2$, the energy density is given by

$$
\mathcal{E}=\sum_{\alpha}\left(\frac{1}{2 m_{\alpha}}\left|\nabla \psi_{\alpha}\right|^{2}+V_{\alpha}^{\mathrm{trap}}\left|\psi_{\alpha}\right|^{2}\right)+\frac{1}{2} \sum_{\alpha \beta} U_{\alpha \beta}\left|\psi_{\alpha}\right|^{2}\left|\psi_{\beta}\right|^{2}
$$

where $m_{\alpha}$ is the atomic mass of species $\alpha$ and the interaction coefficients $U_{\alpha \beta}$ are proportional to the two-body scattering lengths, which we take to be positive, since we consider only repulsive interactions. $V_{\alpha}^{\text {trap }}$ is the trapping potential, which we take to be harmonic $V_{\alpha}^{\text {trap }}=\frac{1}{2} m_{\alpha} \omega_{\alpha}^{2} r^{2}$, where $r$ is the distance from the centre of the trap.

The variation of the energy yields the Gross-Pitaevskii equations

$$
i \frac{\partial \psi_{\alpha}}{\partial t}=\frac{\delta \mathcal{E}}{\delta \bar{\psi}_{\alpha}}=-\frac{1}{2 m_{\alpha}} \nabla^{2} \psi_{\alpha}+U_{\alpha \alpha}\left|\psi_{\alpha}\right|^{2} \psi_{\alpha}+\psi_{\alpha} \sum_{\beta \neq \alpha}\left|\psi_{\beta}\right|^{2}+V_{\alpha}^{\text {trap }} \psi_{\alpha} .
$$

There are several conserved quantities associated with this evolution, and three of these are the energy $E$ and number of atoms of each species $N_{\alpha}$ given by

$$
E=\int \mathcal{E} d^{3} x, \quad N_{\alpha}=\int\left|\psi_{\alpha}\right|^{2} d^{3} x
$$

We shall be concerned with stationary solutions of (2.2. 2 in which have the form $\psi_{\alpha}(\mathbf{x}, t)=$ $e^{-i \mu_{\alpha} t} \Psi_{\alpha}(\mathbf{x})$ where the positive real parameters $\mu_{\alpha}$ are the chemical potentials. Substituting this stationary ansatz into $(2.2)$ produces a static equation for $\Psi_{\alpha}$ (which we shall rename $\psi_{\alpha}$ from now on, since we can restrict to discussing the solution at $t=0$ as the time evolution is simply through the constant phase rotation). The static equations for $\psi_{\alpha}$ are the variational equations which arise as the critical points of the modified energy

$$
\widetilde{E}=E-\sum_{\alpha} \int \mu_{\alpha}\left|\psi_{\alpha}\right|^{2} d^{3} x
$$

This implies that a solution $\psi_{\alpha}$ may also be obtained by minimization of the energy (2.11) subject to the constraint of fixed values of $N_{\alpha}$, with $\mu_{\alpha}$ the associated Lagrange multipliers.

\section{A scaling argument}

In three space dimensions a classical field theory with only scalar fields and no higher derivative (Skyrme) terms in the energy generally does not have topological soliton solutions

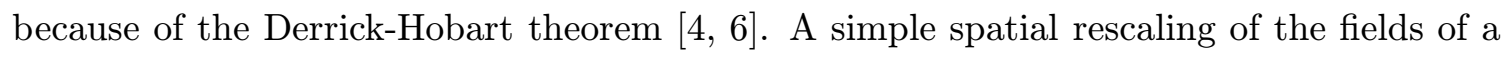


candidate soliton configuration reduces its energy, so there is no local minimum with a finite soliton scale. Here, we give a simple explanation of how the system under consideration evades the Derrick-Hobart theorem, and hence can have soliton solutions.

Decompose the energy $E$ (the integral of $\left(\overline{2} \overline{2} . \overline{1}\left(\begin{array}{l}1 \\ 2\end{array}\right)\right)$ as

$$
E=E_{\text {grad }}+E_{\text {trap }}+E_{\text {int }}
$$

where the three terms denote the contribution to the energy of the gradient terms, the trapping potential and the interaction terms respectively. We wish to consider the effect on this energy of a spatial rescaling $\mathbf{x} \mapsto \lambda \mathbf{x}$. However, the crucial observation is that the energy must be minimized with respect to fixed values for the total number of atoms, $N_{\alpha}$ as defined in $(\overline{2} . \overline{3}$ ij). Thus a simple rescaling is not a valid transformation, as it would change the values of $N_{\alpha}$, unless it is accompanied by the field transformation $\psi_{\alpha} \mapsto \psi_{\alpha} \lambda^{-3 / 2}$. Under these combined scalings the energy transforms as

$$
E \mapsto \frac{1}{\lambda^{2}} E_{\text {grad }}+\lambda^{2} E_{\text {trap }}+\frac{1}{\lambda^{3}} E_{\text {int }} .
$$

This shows that there is no need for a Skyrme term. Furthermore, without the trapping potential the energy can be reduced by expanding a configuration, which is of course the expected behaviour for a system of repulsive atoms. With a trapping potential the energy ( $(\overline{3} \cdot \overline{2})$ contains terms which scale with both positive and negative powers of $\lambda$, thus allowing a minimum at a finite non-zero scale. This demonstrates that a simple scaling transformation can not be used to rule out the possibility of topological solitons, though it does not prove that, should they exist, they will be stable against collapse, since the form of the collapse may be more complicated than the simple rescaling assumed above.

\section{Skyrmion topology}

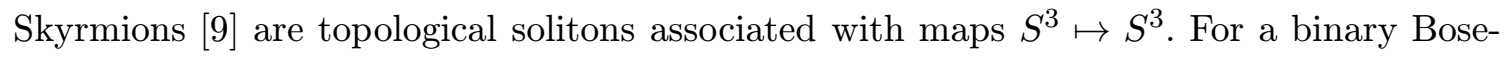
Einstein condensate there are two complex scalar fields so they comprise a map $\left(\psi_{1}, \psi_{2}\right)$ : $\mathbb{R}^{3} \mapsto \mathbb{R}^{4}$, which has no topological structure, and hence naively no topological solitons. However, as we now describe, the topology can enter in a more subtle way.

First, consider a trap loaded with species one. Deep inside the centre of this system, say inside the ball $r \leq L$, the density of species one, $\rho_{1}=\left|\psi_{1}\right|^{2}$, will be approximately constant, falling to zero density far outside the ball $r \gg L$. Next we introduce the second species, but with $N_{1} \gg N_{2}$ so that $\psi_{2}$ is non-zero only near the centre of the ball, and is identically zero at the surface of the ball and outside it. The region in which we are interested is the interior of the ball, so we may restrict our attention to this domain with the boundary condition

$$
\left.\left(\psi_{1}, \psi_{2}\right)\right|_{r=L}=\left(\sqrt{\rho_{1}}, 0\right) .
$$

The phase of $\psi_{1}$ is fixed on the boundary of the ball by energetic considerations and there is no loss of generality in choosing $\psi_{1}$ to be real there. The interior of a 3-ball with all points on the surface identified is topologically $S^{3}$ and hence we have identified the domain

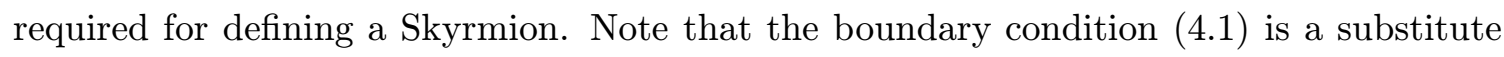


for the role played by the trapping potential for species one, so that from now on we may set $\omega_{1}=0$.

The submanifold of $\mathbb{R}^{4}$ consisting of all points that are a fixed positive distance from the origin obviously yields an $S^{3}$. Under the assumption that the total number density, $\left|\psi_{1}\right|^{2}+\left|\psi_{2}\right|^{2}$, is fixed then the target space is restricted to this $S^{3}$. This assumption was made in [2] and when combined with the above argument we have identified the required map $S^{3} \mapsto S^{3}$. In fact to define this map between 3 -spheres requires a much weaker assumption, namely that the total density never vanishes. It is convenient to identify $S^{3}$ as the group manifold of $S U(2)$ and define the $S U(2)$-valued matrix

$$
M=\frac{1}{2 \sqrt{\left|\psi_{1}\right|^{2}+\left|\psi_{2}\right|^{2}}}\left(\left(\psi_{1}+\bar{\psi}_{1}\right) 1_{2}+\left(\psi_{1}-\bar{\psi}_{1}\right) \sigma_{3}+i\left(\psi_{2}+\bar{\psi}_{2}\right) \sigma_{2}+\left(\psi_{2}-\bar{\psi}_{2}\right) \sigma_{1}\right)
$$

where $1_{2}$ denotes the $2 \times 2$ identity matrix and $\sigma_{i}$ are the Pauli matrices. Clearly this field is well-defined if the total density is non-zero and defines the map $M: S^{3} \mapsto S^{3}$ where the domain is the ball $r \leq L$ and the boundary condition is $M(r=L)=1_{2}$. Due to the homotopy group relation $\pi_{3}\left(S^{3}\right)=\mathbb{Z}$, maps between 3 -spheres are classified by an integer, the Skyrmion number, given explicitly in terms of the field $M$ by the integral

$$
Q=\frac{1}{24 \pi^{2}} \int_{r \leq L} \epsilon_{i j k} \operatorname{Tr}\left(\left(\nabla_{i} M\right) M^{-1}\left(\nabla_{j} M\right) M^{-1}\left(\nabla_{k} M\right) M^{-1}\right) d^{3} x .
$$

Although this topological charge is integer-valued it is not necessarily a conserved quantity since the evolution may produce a point inside the 3 -ball at which the total density vanishes, hence $M$ and $Q$ are not well-defined at this point and time respectively, allowing the configuration to unwind and $Q$ to jump by integer values. In [i-2] it was pointed out that if all the interaction coefficients $U_{\alpha \beta}$ are approximately the same and the solution varies on a length scale much larger than the healing length $\xi=1 / \sqrt{2 m_{1} \rho_{1} U_{11}}$ then energetically it is unfavourable for large variations in the total number density to arise. Thus, it is a good approximation to assume that the total number density is constant, and in particular this rules out the possibility that it might vanish. In this paper we test this approximation by unfreezing the degree of freeedom associated with the total number density. The numerical results of Section iti. confirm that it is a good approximation for the regime we consider, with the total density varying by the order of only one percent over the entire domain, and hence it is certainly non-zero.

\section{Skyrmions as vortons}

In this Section we consider the structure of a Skyrmion by describing a simple $Q=1$ configuration in terms of its constituent fields $\psi_{\alpha}$. The simplest $Q=1$ configuration has the hedgehog form

$$
M=\cos f 1_{2}+i \frac{\sin f}{r} \mathbf{x} \cdot \boldsymbol{\sigma}
$$

where $f(r)$ is a real profile function, which is monotonically decreasing, and satisfies the boundary conditions that $f(0)=\pi$ and $f(L)=0$. In the Skyrme model $[\overline{9}]$ the single Skyrmion solution has exactly the $S O(3)$ symmetric form $\left(\begin{array}{l}1 \\ 0\end{array}\right)$, in which any spatial 
rotation can be compensated by acting with the global $S O(3)$ symmetry of the model. However, a BEC Skyrmion solution will not be given by such a simple expression since (for generic values of the interaction coefficients) the energy (2, $\left.\overline{2}^{\prime} \mathbf{1}^{\prime}\right)$ does not have an $S O(3)$ global symmetry but only a global $U(1) \times U(1)$ symmetry, associated with independent phase rotations of the fields $\psi_{1}$ and $\psi_{2}$. Nevertheless it is useful to examine the configuration (5i) in terms of the fields $\psi_{\alpha}$ as it gives an insight into the composition of a Skyrmion which is topologically correct.

A comparison of $\left(\overline{5} \cdot \overline{1}_{1}\right)$ and $\left(\overline{4} .2_{1}\right)$ shows that $\psi_{1}$ vanishes on a circle in the plane $x_{3}=0$, with a radius $r_{*}$, where this is the unique value such that $f\left(r_{*}\right)=\pi / 2$. Therefore there is a vortex ring in component one. The core of this vortex ring is filled with component two, that is, $\psi_{2} \neq 0$, and moreover the phase of $\psi_{2}$ rotates by $2 \pi$ as one moves around the vortex ring once. It is this twisting of the second component that provides a force to balance the expected contraction of a vortex ring due to its tension. The vortex ring in component one is threaded by a vortex string in component two, which lies along the $x_{3}$-axis, where $\psi_{2}=0$. The core of this vortex string is filled with component one and the phase of $\psi_{1}$ twists by $2 \pi$ along the string.

In terms of this vortex description the Skyrmion has obvious similarities with the cosmic vorton [3;] $\left[\begin{array}{l}3 \\ 1\end{array}\right]$ Vortons are spinning vortex rings in $U(1) \times U(1)$ models that may have formed in phase transitions in the early universe and are candidates for dark matter. Vortons arise in models with both local and global $U(1) \times U(1)$ symmetries and the salient feature is that the potential term in the Lagrangian has the form

$$
V=\lambda_{1}\left(\left|\psi_{1}\right|^{2}-v_{1}^{2}\right)^{2}+\lambda_{2}\left(\left|\psi_{2}\right|^{2}-v_{2}^{2}\right)^{2}+\beta\left|\psi_{1}\right|^{2}\left|\psi_{2}\right|^{2}
$$

where the parameters are chosen such that in the vacuum there is a spontaneous symmetry breaking $U(1) \times U(1) \mapsto U(1)$. Ignoring the gradient and trapping terms this is exactly the

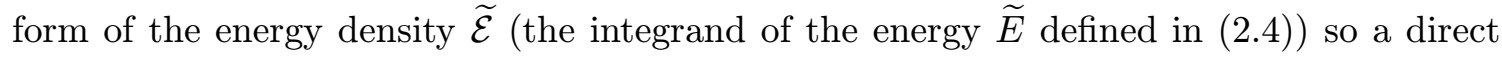
mapping can be established between the parameters of the vorton model and those of the BEC. In the following Section we shall adapt the techniques used to study vorton stability [1] i i] in order to determine parameter regimes in which stable Skyrmion solutions exist.

In this Section we have shown how a BEC Skyrmion may equally well be viewed as a vorton, and this may be a useful perspective in providing a further interesting link between low-temperature laboratory experiments and cosmology.

\section{Phase separation and stability}

In [ī] it was shown that a crucial property of a binary BEC in order to allow stable Skyrmion solutions to exist was that of phase separation. In this Section we rederive this result from a different perspective.

Phase separation is the tendency for a two-component condensate to arrange into regions in space in which the density of one component is high only if the density of the other component is low. Phase separation occurs if the interaction coefficients satisfy the relation $U_{12}^{2}>U_{11} U_{22}$, as is easily derived below. 
Ignoring the gradient and trapping terms the energy density, including the chemical potential terms, is given by

$$
V=\frac{U_{11}}{2}\left|\psi_{1}\right|^{4}+U_{12}\left|\psi_{1}\right|^{2}\left|\psi_{2}\right|^{2}+\frac{U_{22}}{2}\left|\psi_{2}\right|^{4}-\mu_{1}\left|\psi_{1}\right|^{2}-\mu_{2}\left|\psi_{2}\right|^{2}
$$

Critical points of this potential satisfy

$$
\frac{\partial V}{\partial\left|\psi_{1}\right|^{2}}=U_{11}\left|\psi_{1}\right|^{2}+U_{12}\left|\psi_{2}\right|^{2}-\mu_{1}=0 \text { and } \frac{\partial V}{\partial\left|\psi_{2}\right|^{2}}=U_{22}\left|\psi_{2}\right|^{2}+U_{12}\left|\psi_{1}\right|^{2}-\mu_{2}=0
$$

but the determinant of the Hessian is

$$
\operatorname{det} \frac{\partial^{2} V}{\partial\left|\psi_{\alpha}\right|^{2} \partial\left|\psi_{\beta}\right|^{2}}=\operatorname{det}\left(\begin{array}{cc}
U_{11} & U_{12} \\
U_{12} & U_{22}
\end{array}\right)=U_{11} U_{22}-U_{12}^{2}
$$

Thus if $U_{12}^{2}>U_{11} U_{22}$ then the Hessian has a negative eigenvalue and the critical points with $\left|\psi_{1}\right|^{2}>0$ and $\left|\psi_{2}\right|^{2}>0$ are unstable. The stable minima are therefore obtained at the boundary of the domain of the variables $\left|\psi_{1}\right|^{2}$ and $\left|\psi_{2}\right|^{2}$, that is, where $\left|\psi_{1}\right|^{2}\left|\psi_{2}\right|^{2}=0$. In other words the stable minima are where one or other of the components vanish and there is phase separation. Obviously this simple picture of regions where one or other of the components vanishes identically is an approximation based on the fact that the gradient terms have been neglected, but the general picture of regions where the density of only one component is high remains valid after the gradient energy smoothing.

Let us rewrite the potential term ( $\left(\overline{6} \cdot \overline{6}_{i}^{\prime}\right)$ as

$$
V=\frac{1}{2} U_{11}\left(\left|\psi_{1}\right|^{2}-\frac{\mu_{1}}{U_{11}}\right)^{2}+\frac{1}{2} U_{22}\left(\left|\psi_{2}\right|^{2}-\frac{\mu_{2}}{U_{22}}\right)^{2}+U_{21}\left|\psi_{1}\right|^{2}\left|\psi_{2}\right|^{2}-\frac{\mu_{1}^{2}}{2 U_{11}}-\frac{\mu_{2}^{2}}{2 U_{22}}
$$

which makes it clear that it has the vorton form (1). If we think in terms of the vorton description of a Skyrmion then we can easily adapt the stability analysis used for vortons

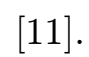

In the vortex string along the $x_{3}$-axis we have $\psi_{2}=0$, and hence $\left|\psi_{1}\right|^{2}=\frac{\mu_{1}}{U_{11}}$. Since we require $\psi_{2}=0$ to be a stable solution in this background this means that the effective mass of the $\psi_{2}$ field must be positive

$$
\frac{1}{2} m_{e f f}^{2}=-\mu_{2}+U_{12} \frac{\mu_{1}}{U_{11}}>0
$$

Similarly in the core of the vortex ring where $\psi_{1}=0$ we have $\left|\psi_{2}\right|^{2}=\frac{\mu_{2}}{U_{22}}$ and for stability the effective mass of the $\psi_{2}$ field must be positive in this background

$$
\frac{1}{2} \tilde{m}_{e f f}^{2}=-\mu_{1}+U_{12} \frac{\mu_{2}}{U_{22}}>0 .
$$

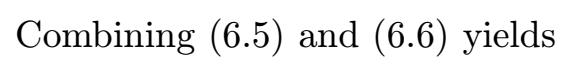

$$
\frac{U_{12}}{U_{11}}>\frac{\mu_{2}}{\mu_{1}}>\frac{U_{22}}{U_{12}}
$$


which in particular implies that there is phase separation

$$
U_{12}^{2}>U_{11} U_{22} \text {. }
$$

Obviously the above argument relies on an idealized description of the vorton and should not be treated as a rigorous result but rather as an approximate condition. Attempts at estimating the corrections due to the neglected effects suggest that the phase separation relation ( $\left({ }_{1}^{6} \overline{6} \overline{8}_{i}^{i}\right)$ is a necessary but not sufficient criterion to obtain a Skyrmion solution. This explains why the earlier study [is] failed to find a solution, since it made the assumption that all the interaction coefficients were exactly equal - so there was no phase separation.

There are physically realizable BECs which do satisfy the phase separation condition (6.6.-1). In principle, two-component BECs could be formed with each component representing a different atomic species, but in practice current experiments have been performed in which the two components represent two different hyperfine levels of the same atomic species. The system ${ }^{87} \mathrm{Rb}\left|F=2, m_{F}=1\right\rangle|1,-1\rangle$ has been studied experimentally and phase separation observed $\left[\begin{array}{l}5-5 \\ \text {. }\end{array}\right.$. For this system the interaction coefficients are approximately equal, but more precisely have the relative ratios

$$
U_{11}: U_{12}: U_{22}=0.94: 0.97: 1.00
$$

so that $U_{12}^{2}=1.00096 U_{11} U_{22}>U_{11} U_{22}$, confirming phase separation. In the following Section we discuss some numerical results using these values.

\section{Numerical results}

The numerical approach used is to minimize the energy $(\overline{2}-\overline{4}-4)$ using a simple finite difference discretization on a grid containing $101^{3}$ points and a gradient flow algorithm for the relaxation. The initial condition is taken to be of the hedgehog form $\left(\overline{5} . \bar{j}_{1}^{1}\right)$ with a suitable profile function $f(r)$. Our simulation will be confined to the region $\left|x_{i}\right| \leq L$, for some $L$, this being the numerical analogue of the earlier theoretical 3-ball $r \leq L$ outside which the density of component one begins to decay to zero. The numerical implementation of the boundary condition ( $(\overline{1}-1.1)$ is therefore to fix $\left(\psi_{1}, \psi_{2}\right)=\left(\sqrt{\rho_{1}}, 0\right)$ on the boundary of the grid with $\rho_{1}=\mu_{1} / U_{11}$ the appropriate constant density. We keep the experimentally relevant ratios $\left(\overline{6}, \bar{g}_{i}^{i}\right)$ but choose all other parameter values for convenience. Explicitly we set $\mu_{1}=U_{11}$ so that $\psi_{1}=1$ on the boundary of the grid, $m_{1}=m_{2}=0.5, \omega_{2}=0.01$, $L=50$, and $U_{22}=200$. The remaining free parameter is $\mu_{2}$, which determines the number of atoms of component two. In choosing this value recall the inequality $\left(\overline{6} . \overline{6} \overline{7}_{j}\right)$, which for the experimental parameters $(6.9 .9)$ determines the range $1.0309<\mu_{2} / \mu_{1}<1.0319$. This is an extremely narrow range for $\mu_{2}$, which would be difficult to find by trial and error. The numerical results described below are for the value $\mu_{2} / \mu_{1}=1.0318$, which is within the required range and produces good results. Values tried which were outside the above range did not produce Skyrmion solutions. Note that it may seem that the fine tuning of $\mu_{2}$ might make it difficult to construct Skyrmions experimentally. However, this is not the case since the variable under experimental control is the number of atoms $N_{2}$, which 
turns out to be highly sensitive to changes in $\mu_{2}$. Thus a large range of values of $N_{2}$ can be obtained, and in fact it appears that a more convenient approach is the minimization of the energy subject to a constraint on $N_{2}$ itself, rather than employing the chemical potential $\mu_{2}$. With this numerical approach it is also possible to dispense with the trapping of the second component and set $\omega_{2}=0$. This shows that the trap is not an important feature in the study of Skyrmions in BECs.

The numerical results for $\mu_{2}=1.0318 \mu_{1}$ are displayed in fig. 1 . In fig. ili (a) we display
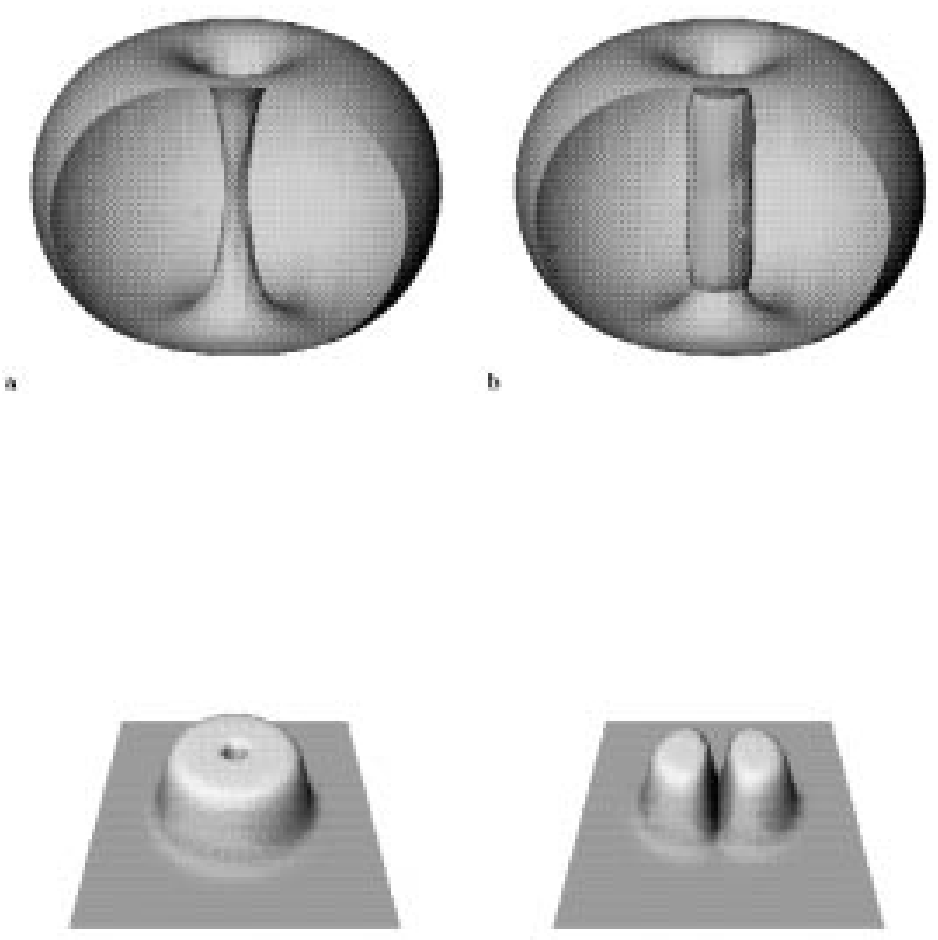

Figure 1: The $Q=1$ Skyrmion. (a) The isosurface $\left|\psi_{2}\right|^{2}=0.5$, with a section cut to view the interior. (b) As (a) but including an isosurface of topological charge density. (c) $\left|\psi_{2}\right|^{2}$ in the plane $x_{3}=0$. (d) $\left|\psi_{2}\right|^{2}$ in the plane $x_{2}=0$.

the isosurface $\left|\psi_{2}\right|^{2}=0.5$, with a section cut out so that the interior structure is visible. The vortex string can clearly be seen as the tube along the $x_{3}$-axis. The region surrounding the tube and inside the shell is where the second condensate is large and is associated with the core of the vortex ring. In fig. ${ }_{1}^{1}$ (b) we reproduce the isosurface in (a) but overlay a surface of constant topological charge density (the integrand in equation $(\overline{4} . \overline{3}$ i) ). This shows that the baryon density, whose integration produces the value one to within a reasonable numerical accuracy, is highly localized around the vortex tube along the $x_{3}$-axis, corresponding to a boundary between two phase separated regions. This distribution of the topological charge density is very different to that in the Skyrme model, where it is spherically symmetric and decays with the distance from the origin, and demonstrates that although the topological 
features are similar for Skyrmions in BECs the qualitative details are very different. In fig. ${ }_{1}^{1}(\mathrm{c})$ we plot the density of component two $\left|\psi_{2}\right|^{2}$ in the plane $x_{3}=0$, which contains the vortex ring, and in fig. $]_{1}^{1}(\mathrm{~d})$ we plot the same quantity in the plane $x_{2}=0$, which reveals the vortex anti-vortex pair associated with slicing the ring in a plane containing the symmetry axis.

\section{Conclusion}

We have described some aspects of Skyrmions in binary BECs, focusing on finding parameter regimes in which stable Skyrmions exist. Appropriate conditions have been identified and tested by a numerical solution of the Gross-Pitaevskii equations. It would clearly be very interesting if Skyrmions could be created experimentally in BECs and in this direction future numerical work on the dynamical creation of Skyrmions, extending the initial investigations of [i] to regimes with Skyrmion solutions, would be worthwhile. Other aspects to be studied include examining how the properties of a Skyrmion change with its angular momentum and the structure of multi-Skyrmion solutions.

\section{Acknowledgments}

We thank the EPSRC (PMS) and PPARC (RAB) for advanced fellowships.

\section{References}

[1] U. Al Khawaja and H. Stoof, №ture

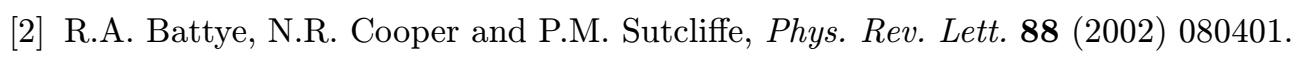

[3] R.L. Davis and E.P.S. Shellard, Nucl. Phys. B $\mathbf{3 2} \overline{3}(19 \overline{9} \overline{9}) 209$.

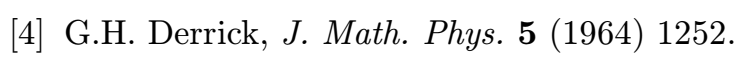

[5] D.S. Hall et al., Phys. Rev. Lett. 81 (1998) 1539.

[6] R. Hobart, Troc. Phys. Soc. London 82 (1963) 201 i.

[7] M.R. Matthews, B.P. Anderson, P.C. Haljan, D.S. Hall, C.E. Wieman and E.A. Cornell, Phys. Rev. Lett. 83 $(19999)-2498$

[8] J. Ruostekoski and J.R. Anglin, Physs. Rev. Lett. 86 (2001) 39334.

[9] T.H.R. Skyrme, 'Proc. Roy. Soc. Á260 (1961) 127 ;

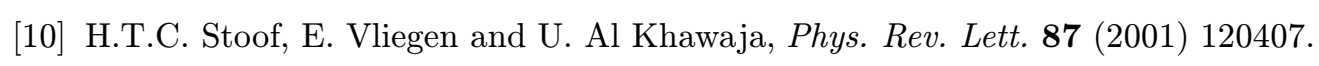

[11] A. Vilenkin and E.P.S. Shellard, Cosmic strings and other topological defects, Cambridge University Press 1994. 